\title{
Effects of a Chemically Synthesized Leucine-Rich Amelogenin Peptide (csLRAP) on Chondrogenic and Osteogenic Cells
}

\author{
Yuko Matsuda' $^{1)}$, Yuji Hatakeyama ${ }^{2) *}$, Kazuki Nakashima ${ }^{1)}$, Naoko Kamogashira1), Junko Hatakeyama ${ }^{3)}$, \\ Sachio Tamaoki'), Yoshihiko Sawa ${ }^{2)}$ and Hiroyuki Ishikawa ${ }^{1)}$. \\ 1) Section of Orthodontics, Fukuoka Dental College, Fukuoka, Japan \\ 2) Section of Functional Structure, Fukuoka Dental College, Fukuoka, Japan \\ 3) Section of Operative Dentistry and Endodontology, Fukuoka Dental College, Fukuoka, Japan \\ (Accepted for publication, October 26, 2016)
}

\begin{abstract}
Amelogenin is one of the enamel matrices secreted by ameloblasts and is known to have at least 15 alternative mRNA splicing isoforms. The leucine rich amelogenin peptide (LRAP) is one of most abundant amelogenin isoforms and numerous studies have shown that amelogenin peptides including LRAP, are expressed in cartilage and bone. Lysosome-associated membrane protein-1 (LAMP-1) has been identified as amelogenin binding partner, however, the mechanism of action for LRAP in chondrogenesis or osteogenesis is still unclear. This study aimed to assay the effect of chemically synthesized LRAP (csLRAP) on chondrogenic or osteogenic differentiation with the involvement of LAMP-1. The csLRAP used in this study was generated by F-moc solid phase chemistry based on the mouse LRAP amino acid sequence and was added to the culture medium of the chondrogenic cells, ATDC5 and the osteoblast cells, MC3T3-E1. Both chondrogenic and osteoblast cells, in which an immunopositive reaction to LAMP-1 antibody was observed by immunocytochemistry, showed significant suppression in cell number in the presence of csLRAP at $10 \mu \mathrm{g} / \mathrm{ml} \mathrm{compared} \mathrm{to} \mathrm{that} \mathrm{in} \mathrm{control,} \mathrm{but}$ this effect was rescued in the presence of LAMP-1 antibody. On the other hand, the intensity of alcian blue staining in chondrogenic cells and alizarin red staining in osteoblast cells was significantly increased in the presence of csLRAP at a dose of $10 \mu \mathrm{g} / \mathrm{ml}$ after 4 weeks culture, and this effect was suppressed in the presence LAMP-1 antibody. Moreover, the chondrogenic or osteogenic differentiation marker genes including Sox9, Type II Collagen, Type X Collagen, Runx2, Alkaline Phosphatase, and Type I Collagen, were upregulated in the presence of csLRAP after one week of culture and were suppressed in the presence of LAMP-1 antibody. These results suggest that csLRAP could promote osteogenic and chondrogenic differentiation in vitro, and that LAMP-1 may be involved in the differentiation and proliferation of these cells.

Key words: Chondrogenesis, Leucine-rich amelogenin peptide (LRAP), Lysosome-associated membrane proteins (LAMPs), Osteogenesis
\end{abstract}

\section{Introduction}

Ameloblasts secrete enamel matrix proteins during dental enamel formation and amelogenin is the most abundant enamel matrix; mutation in amelogenin causes amelogenesis imperfecta in humans and mice. In mice, ameloblasts generate at least 15 alternative mRNA splicing isoforms of amelogenin encompassing exon 1 to exon $9^{1,2)}$, which encode various lengths of amino acid sequences. M180 is most abundant amelogenin isoform, in which the exon 4 is excluded from the full-length transcript of M194. The leucine-rich amelogenin peptide (LRAP) is one of two most abundant amelogenin isoforms, and was first proposed to explain the presence of alternative splice isoforms in amelogenin ${ }^{1)}$ and is

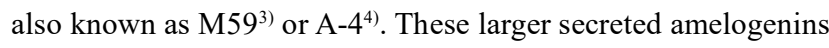

Correspondence to: Dr. Yuji Hatakeyama, Department of Morphological Biology, Fukuoka Dental College, 2-15-1 Tamura, Sawara-ku, Fukuoka, 814-0193 Japan; Tel: +81-92-801-0411; Fax: +81-92-801-4909; E-mail: hatakeyy@college.fdcnet.ac.jp are degraded into various smaller amelogenin peptides by proteinases such as matrix metalloproteinase-20 (MMP-20) and kallikrein 4 (KLK4) during enamel mineralization ${ }^{5)}$ and organic matrices including amelogenin in the enamel are almost completely removed during enamel mineralization before tooth eruption.

In this decade, several studies have revealed that amelogenin isoform genes and proteins are expressed not only in enamel formation but also in adult connective tissue forming cells like rat osteoblasts $^{6}$, , rat chondrocytes ${ }^{6}$, odontoblasts $^{7)}$, cementoblasts ${ }^{8,9)}$ and periodontal ligament cells ${ }^{8,10)}$. In amelogenin null mice, dramatically reduced expression of bone sialoprotein (BSP), a mineralized tissue-specific marker, was observed at both the gene and protein levels ${ }^{11)}$. Furthermore, a number of in vitro studies show that amelogenin is involved in osteo-chondrogenesis of various species or in different developmental stages of mesenchymal cells like embryonic mouse muscle fibroblasts ${ }^{4}$, mouse embryonic stem cells ${ }^{13)}$, mouse chondrogenic ${ }^{14)}$ and 
osteoblastic cells ${ }^{15)}$, and primary human articular cartilage cells ${ }^{16}$. As we have reported previously, LRAP promotes chondrogenesis in mouse limb mesenchymal cells ${ }^{16)}$. These reports indicate that amelogenin isoforms may have effects that include signaling molecules in differentiation of cells derived from the mesenchyme.

Lysosomes are cellular organelles that are involved in endocytosis, phagocytosis, and autophagy. Two classes of proteins are essential for lysosomal function: acid hydrolases and lysosomal membrane proteins. The most abundant lysosomal membrane proteins are lysosome-associated membrane protein-1 (LAMP-1, CD107a), LAMP-2 (CD107b), and LAMP-3 (CD63). LAMPs are not only present in the lysosome membrane but are also present in endosomes, phagosomes, and cell surfaces ${ }^{17)}$. Since LAMP-1 and LAMP-3 are identified as binding partners for mouse amelogenin ${ }^{18,19)}$, several studies have reported that LAMP-1 expressed on the cell membrane binds to amelogenin and could be a signaling receptor for amelogenin ${ }^{20,21)}$. However, the detailed mechanism by which the amelogenin isoform induces chondrogenesis or osteogenesis is still unclear. In previous reports, amelogenin isoforms were generated from $E$. $\operatorname{coli}^{4,16)}$ and from mammalian cells ${ }^{13)}$. Since solid phase protein synthesis allows higher purity and prevents toxic materials compared to the process using biological methods like bacteria ${ }^{22)}$, the purpose of this study is to evaluate the biological effect of chemically synthesized LRAP (csLRAP) on chondrogenesis and osteogenesis in vitro and to clarify the role of LAMP-1 in chondrogenic or osteogenic cell differentiation induced by csLRAP.

\section{Materials and Methods \\ Cell culture and chemical synthesis of LRAP}

The mouse chondrogenic cell line ATDC5 and osteoblast cell line MC3T3-E1 were obtained from the Riken Bioresource Center Cell Bank (Tsukuba, Japan). The ATDC5 and MC3T3-E1 cells were cultured to an appropriate confluence for each experiment using respective growth medium. ATDC5 cells were grown in a medium consisting of 1:1 mixture of Dulbecco's Modified Eagle Medium (D-MEM) and Ham's F12 (Invitrogen/Life Technologies, Tokyo, Japan), with 5\% fetal bovine serum (FBS, Equitech-Bio Inc., Tokyo, Japan), $3 \times 10^{-8} \mathrm{M}$ sodium selenite (Wako, Osaka, Japan), and $10 \mu \mathrm{g} / \mathrm{ml}$ holo-form bovine transferrin (Invitrogen). MC3T3-E1 cells were cultured in $\alpha$-MEM (Invitrogen/Life Technologies, Tokyo, Japan) with 10\% FBS. Both growth media included antibiotics $(100 \mathrm{U} / \mathrm{ml}$ penicillin-G and $100 \mathrm{mg} / \mathrm{ml}$ streptomycin) and both cells were cultured at $37^{\circ} \mathrm{C}$ in a humidified atmosphere with $5 \% \mathrm{CO}_{2}$ in air. We purchased LRAP synthesized using F-moc (9-fluorenylmethoxycarbonyl) solid phase chemistry from BEX Co., Ltd. (Tokyo) and the mouse LRAP amino acid sequence used in this study was obtained from a previous report ${ }^{23)}$. LRAP was dissolved in dimethyl sulfoxide (DMSO) to $10 \mathrm{mg} / \mathrm{ml}$ and was diluted in medium to the appropriate concentration for each experiment.

\section{Cell proliferation}

The cell numbers were counted as described previously ${ }^{24)}$ using a Cell Counting Kit-8 (Dojindo Laboratories, Kumamoto, Japan), which depends on the measurement of a highly water-soluble formazan dye produced from tetrazolium salts. ATDC5 and MC3T3-E1 cells were seeded at $1 \times 10^{3}$ cells per well on a 96well plate, and after 3 hours for initial cell attachment, the serumfree growth medium was changed to media with or without 10 $\mu \mathrm{g} / \mathrm{ml}$ of LRAP. For the inhibition experiment, the medium was changed to contain both LRAP and rat monoclonal antibody to mouse LAMP-1 (Santa Cruz Biotech, Tokyo, Japan) diluted to 2 $\mu \mathrm{g} / \mathrm{ml}$ according to a previous report ${ }^{25)}$. After 48 hours of culture, the cells were incubated with the counting reagent for one hour as per the manufacturer's instructions. The relative cell number was then determined by measuring the absorbance of the formazan dye product in the cultures at a wavelength of $450 \mathrm{~nm}(1420$ Multilabel counter ARVO MS; PerkinElmer, Waltham, MA).

\section{Alcian blue staining and Alizarin Red staining}

ATDC5 and MC3T3-E1 cells were seeded on 12-well plates. After achieving confluence, the medium was changed to chondrogenic differentiation medium (DMEM/F12 supplemented with $2.5 \% \mathrm{FBS}, 5 \mu \mathrm{g} / \mathrm{ml}$ insulin, $5 \mu \mathrm{g} / \mathrm{ml}$ transferrin, and $5 \mathrm{ng} / \mathrm{ml}$ selenous acid (ITS reagent; Becton, Dickinson and Company)) or osteogenic differentiation medium (D-MEM supplemented with $5 \% \mathrm{FBS}, 50 \mu \mathrm{g} / \mathrm{ml}$ ascorbic acid, $10 \mathrm{nM}$ dexamethasone, and 10 $\mathrm{mM} \beta$-glycerophosphate) with $10 \mathrm{mg} / \mathrm{ml}$ LRAP, or LRAP with 2 $\mu \mathrm{g} / \mathrm{ml}$ of anti-LAMP-1 as in the proliferation assay. After 4 weeks of culture in each medium with LRAP or the antibody, which was changed twice per week, ATDC5 cells were subjected to alcian blue staining and MC3T3-E1 cells were subjected to Alizarin Red staining. Alcian blue staining was performed as described previously with minor modifications ${ }^{26)}$. Briefly, cultures were fixed in $2 \%$ acetic acid in ethanol for 15 minutes at room temperature, rehydrated, and stained overnight at room temperature with $0.5 \%$ Alcian Blue Stain Solution at pH 2.5 (Muto Pure Chemicals, Tokyo, Japan). The intensity of alcian blue staining was measured as absorbance at $600 \mathrm{~nm}$ (1420 Multilabel counter ARVO MS, PerkinElmer) after solubilizing the alcian blue stained cartilage matrices in 4M guanidine hydrochloride. For alizarin red staining, MC3T3-E1 cells were fixed in ice-cold 70\% methanol for 20 minutes after 4 weeks of culture. After fixation, the cells were rehydrated with distilled water and stained for 5 minutes with $0.5 \%$ alizarin red S (Sigma-Aldrich, Tokyo Japan). To quantify matrix calcification, $10 \%$ cetylpyridinium chloride was added to the stained cultures and incubated for 10 minutes to release the calcium-bound alizarin red $\mathrm{S}$. The solubilized stains were transferred to a 96-well plate and absorbance at $570 \mathrm{~nm}$ was 

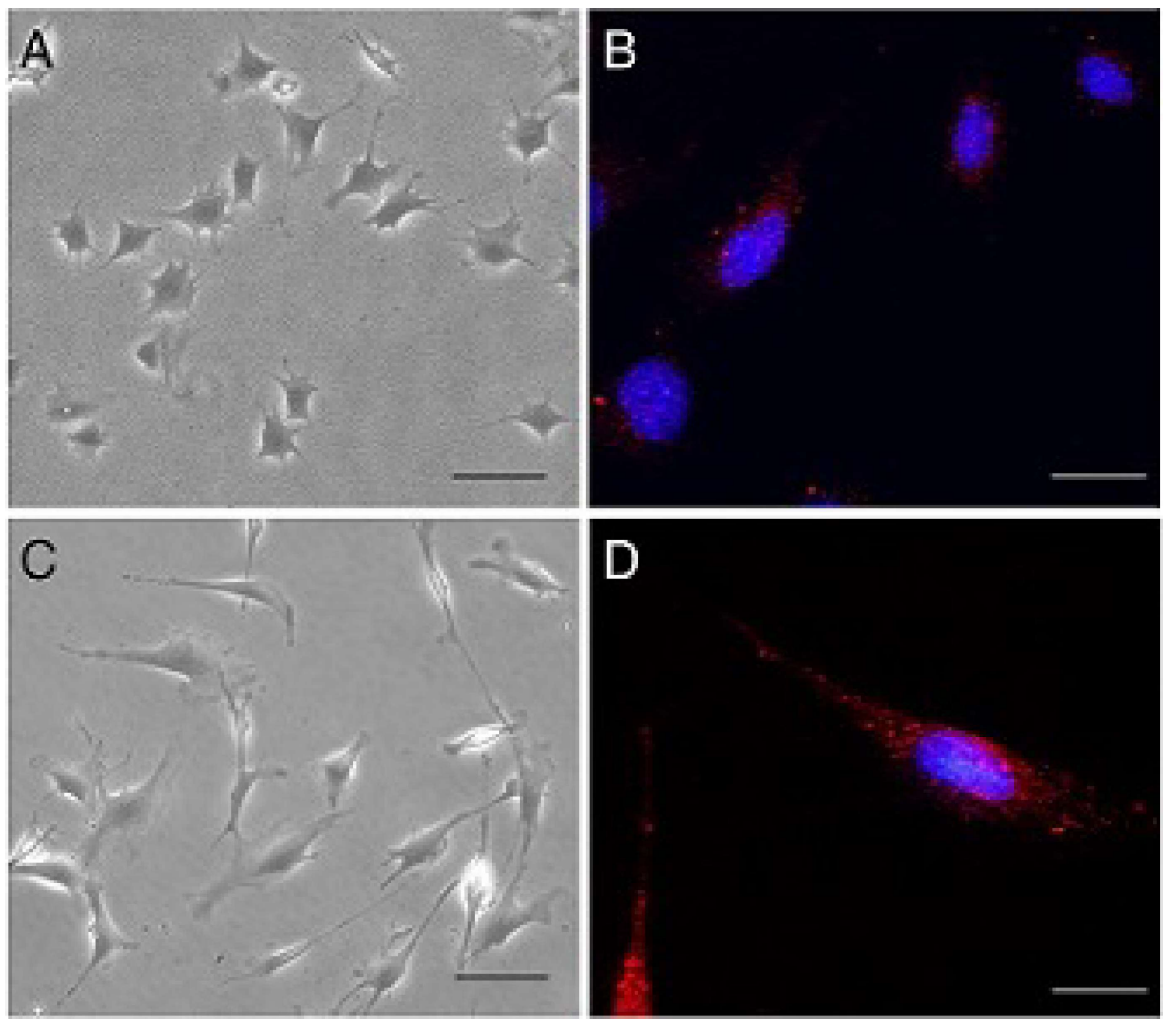

Figure 1. LAMP-1 immunocytochemistry in chondrogenic and osteogenic cells. Cells were seeded on a collagen type I coated glass slide in growth medium at 50\% confluence. After one day of culture, cells were fixed and then stained with anti-mouse LAMP-1 (Red) and DAPI (Blue). ATDC5 (A) cells showed high nuclear-cytoplasmic ratio and MC3T3-E1 (C) cells showed a spindle shaped morphology in phase-contrast microscopy observation. Both ATDC5 (B) and MC3T3-E1 (D) cells expressed LAMP-1. (A and C, Scale bar $=100$ $\mu \mathrm{m}, \mathrm{B}$ and $\mathrm{D}$, Scale bar $=50 \mu \mathrm{m})$

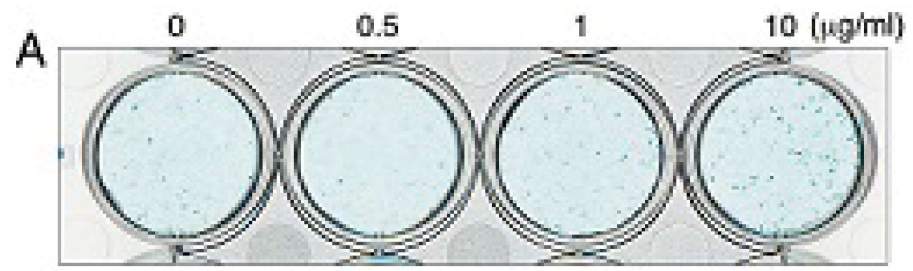

$\mathrm{B}$
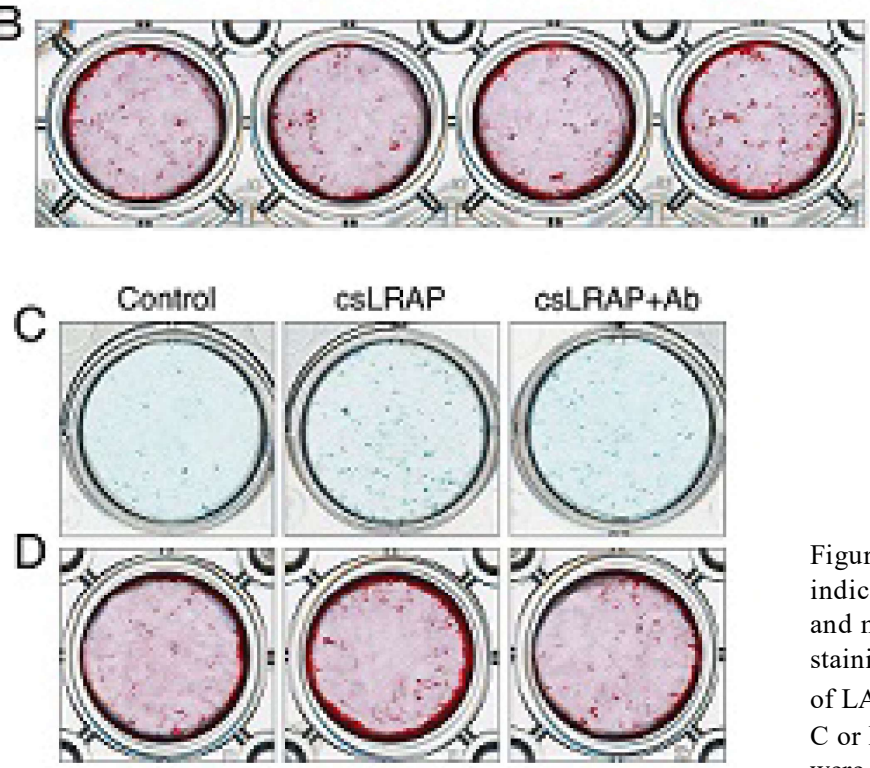

Figure 2. csLRAP promoted chondrogenic matrix formation indicated by alcian blue staining (A) in ATDC5 cell culture and mineralized nodules formation indicated by alizarin red staining (B) in MC3T3 cell culture. In the combined presence of LAMP-1 antibody and csLRAP at $10 \mu \mathrm{g} / \mathrm{ml}$ (csLRAP $+\mathrm{Ab}$, $\mathrm{C}$ or D) alcian blue staining $(\mathrm{C})$ and alizarin red staining (D) were suppressed for 4 weeks culture. 
measured as described in a previous report $\mathrm{t}^{27)}$.

\section{Immunofluorescence assay}

For LAMP-1 immunostaining in ATDC5 and MC3T3, cells were seeded on type I collagen coated glass slides (Iwaki, Tokyo, Japan) in respective growth media to $20 \%$ confluence and after one day of culture, the cells were fixed in $4 \%$ paraformaldehyde in PBS for 10 minutes. After washing with PBS, cells were incubated with rabbit polyclonal anti-mouse LAMP-1 (ab24170, Abcam, Tokyo Japan) diluted to $1 \mu \mathrm{g} / \mathrm{ml}$ in $1 \%$ bovine serum albumin (BSA) for one hour at room temperature. After incubation with the primary antibody, cells were incubated with goat antirabbit Alexa Flour 594 secondary antibody (Thermo Fisher Scientific, Waltham, MA, USA) diluted to 1:500 in 1\% BSA for one hour at room temperature, and diamino-2-phenylindole (DAPI) as used for nuclear counterstaining.

\section{Quantitative Reverse Transcription Polymerase Chain Reaction (real-time quantitative RT-PCR)}

The expression of chondrogenic differentiation marker genes (Sox9, collagen type II (Col II), collagen type X (Col X), and aggrecan) and osteogenic differentiation marker genes (Runx2, Alkaline Phosphatase, liver/bone/kidney (ALP), collagen type I alpha 2 (Colla2), bone sialoprotein (BSP), osteopontin (OPN), and osteocalcin (OCN)) was analyzed by real-time quantitative RT-PCR (MX3005P, Agilent Technologies, Tokyo, Japan). Total RNA was extracted from the ATDC5 and MC3T3-E1 cells after one week of culture after initial plating for 2 hours, using the NucleoSpin RNA kit (Macherey-Nagel GmbH \& So, KG, Germany, Takara Bio Inc., Kusatsu, Japan) according to the manufacturer's instructions, followed by DNase treatment of the samples. Reverse transcription and real time PCR was performed as previously reported with minor modifications ${ }^{28)}$. Briefly, mRNA was reverse-transcribed to cDNA using the iScript ${ }^{\mathrm{TM}}$ RT Supermix for RT-qPCR (Bio-Rad, Tokyo, Japan). Real-time PCR was performed using iTaq ${ }^{\mathrm{TM}}$ SYBR Green Supermix With ROX (Bio$\mathrm{Rad}$ ) for 45 cycles of $95^{\circ} \mathrm{C}$ for 30 seconds, $60^{\circ} \mathrm{C}$ for 45 seconds, and $72^{\circ} \mathrm{C}$ for 30 seconds, using specific primers designed for osteogenic and chondrogenic differentiation marker genes as previously reported ${ }^{13,28-30)}$. Mouse Col1a2 specific primers were designed using National Center for Biotechnology Information (NCBI) Primer-BLAST and their sequences were as follows: Colla2 (NM_007743.3), 52 -AGC GGT GAA GAA GGA AAG and 52 ACC TTT GCC ACC TTG AAC (512bp amplification product). Melting curve analysis was conducted to determine PCR specificity and the threshold cycle (CT) was defined as the fractional cycle number. Gene expression values are expressed as ratios of GAPDH expression values to those of the gene of interest and relative standard curves representing 10-fold serial dilutions of standard cDNA (1:10:100:1000) were used for linear regression analyses of unknown samples as previously reported ${ }^{30)}$.

\section{Statistical Analysis}

All values are reported as mean \pm SD of four or eight samples per group for each experiment. Statistical significance was determined using Kruskal-Wallis one-way analysis of variance (ANOVA) followed by a post-hoc t-test. Differences were considered significant at a $\mathrm{p}$ value of $<0.05$.

\section{Results}

\section{Immunocytochemistry}

To examine the expression of LAMP-1 in the chondrogenic cells ATDC5 and the osteoblast cells MC3T3-E1, we performed immunocytochemistry on these cells. After one day of culture for initial cell attachment on collagen type I coated glass slides, ATDC5 (Fig. 1A) cells showed high nuclear-cytoplasmic ratio and MC3T3-E1 (Fig. 1C) cells showed a spindle shaped morphology in phase-contrast microscopy observation. Both ATDC5 (Fig. 1B) and MC3T3-E1 (Fig. 1D) were found to be positive for LAMP-1 in the entire cytoplasmic and marginal region of the cell without permeabilization.

\section{Alcian blue and alizarin red staining}

To determine the effect of csLRAP on chondrogenic matrix production or mineralized nodule formation, we performed alcian blue staining for chondrogenic cells (Fig. 2A) and alizarin red staining for osteoblast cells (Fig. 2B) after 4 weeks of culture in differentiation medium with or without csLRAP. In chondrogenic cells, the intensity of alcian blue staining showed an increasing tendency in the presence of $1 \mu \mathrm{g} / \mathrm{ml}$ of csLRAP and a significant difference in the presence of $10 \mu \mathrm{g} / \mathrm{ml}$ csLRAP compared to that in the control although no difference was observed with $0.5 \mu \mathrm{g} /$ $\mathrm{ml}$ csLRAP compared to that in the control (Fig. 2A, Fig. 3A). This increasing alcian blue staining at $10 \mu \mathrm{g} / \mathrm{ml}$ csLRAP was suppressed in the combined presence of LAMP-1 antibody and csLRAP (Fig. 2C, Fig. 3C). Similar to chondrogenic cells, the intensity of alizarin red staining in osteoblast cells with $10 \mu \mathrm{g} / \mathrm{ml}$ csLRAP was increased with significantly compared to that in the control (Fig. 2B, Fig. 3B) and was suppressed in the combined presence of LAMP-1 antibody and csLRAP at $10 \mu \mathrm{g} / \mathrm{ml}$ (Fig. 2D, Fig. 3D).

\section{Expression of chondrogenic and osteogenic marker genes}

To investigate the gene expression profiles in chondrogenic or osteogenic cell differentiation promoted by csLRAP, we performed quantitative RT-PCR for each differentiation marker gene in the chondrogenic or osteogenic cell culture. After one week of culture in the presence of $10 \mu \mathrm{g} / \mathrm{ml} \mathrm{csLRAP,} \mathrm{expression}$ of all chondrogenic marker genes including Sox9 (Fig. 4A), Col II (Fig. 4B), Col X (Fig. 4C), and aggrecan (Fig. 4D), was 
Yuko Matsuda et al:: Effects of csLRAP on Chondrogenesis and Osteogenesis
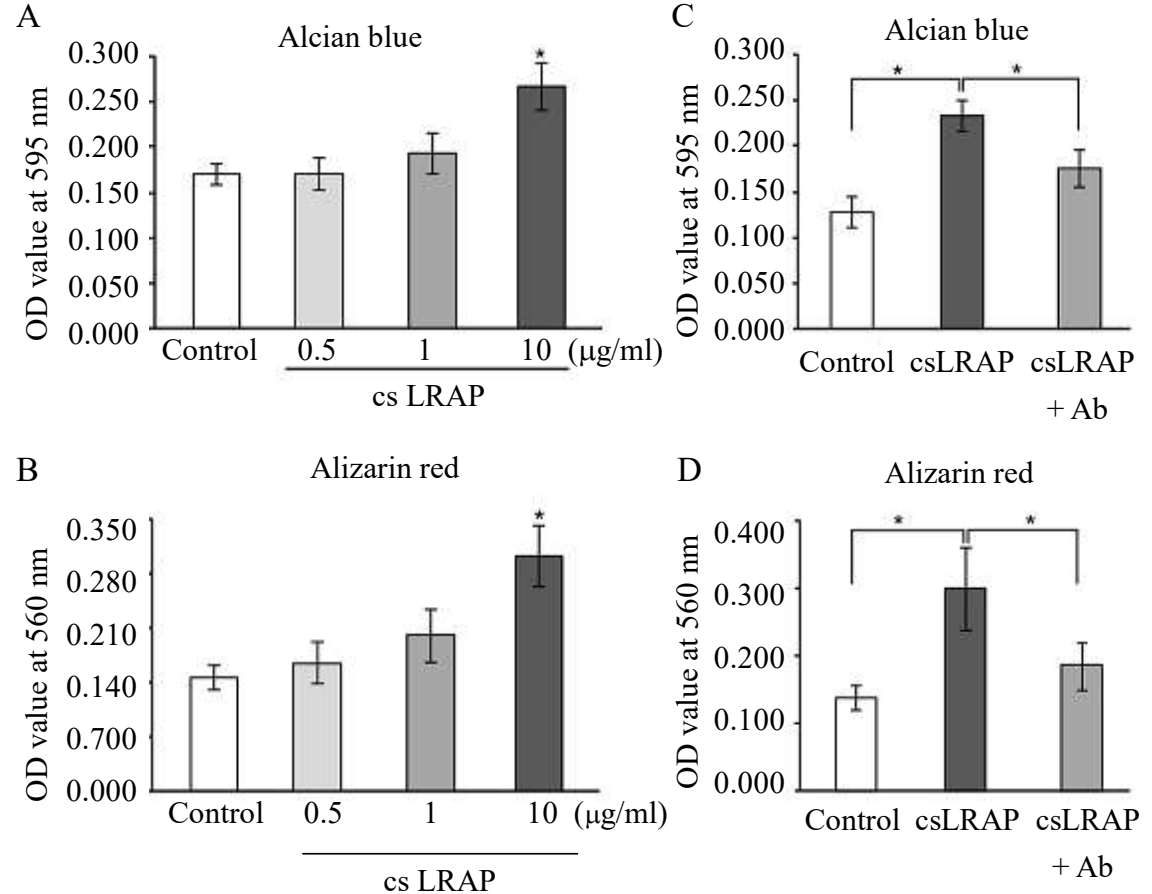

Figure 3. Quantitative analysis of the alcian blue staining and alizarin red staining. The intensity of alcian blue (A) and alizarin red staining (B) in the presence of $10 \mu \mathrm{g} / \mathrm{ml}$ csLRAP was significantly increased compared with that in control. Both the alcian blue (C) and alizarin red staining (D) increased with $10 \mu \mathrm{g} / \mathrm{ml}$ csLRAP was suppressed in the combined presence of LAMP-1 antibody and csLRAP at $10 \mu \mathrm{g} / \mathrm{ml}$ (csLRAP $+\mathrm{Ab}, \mathrm{C}$ or D). $n=8,{ }^{*} p<0.05$
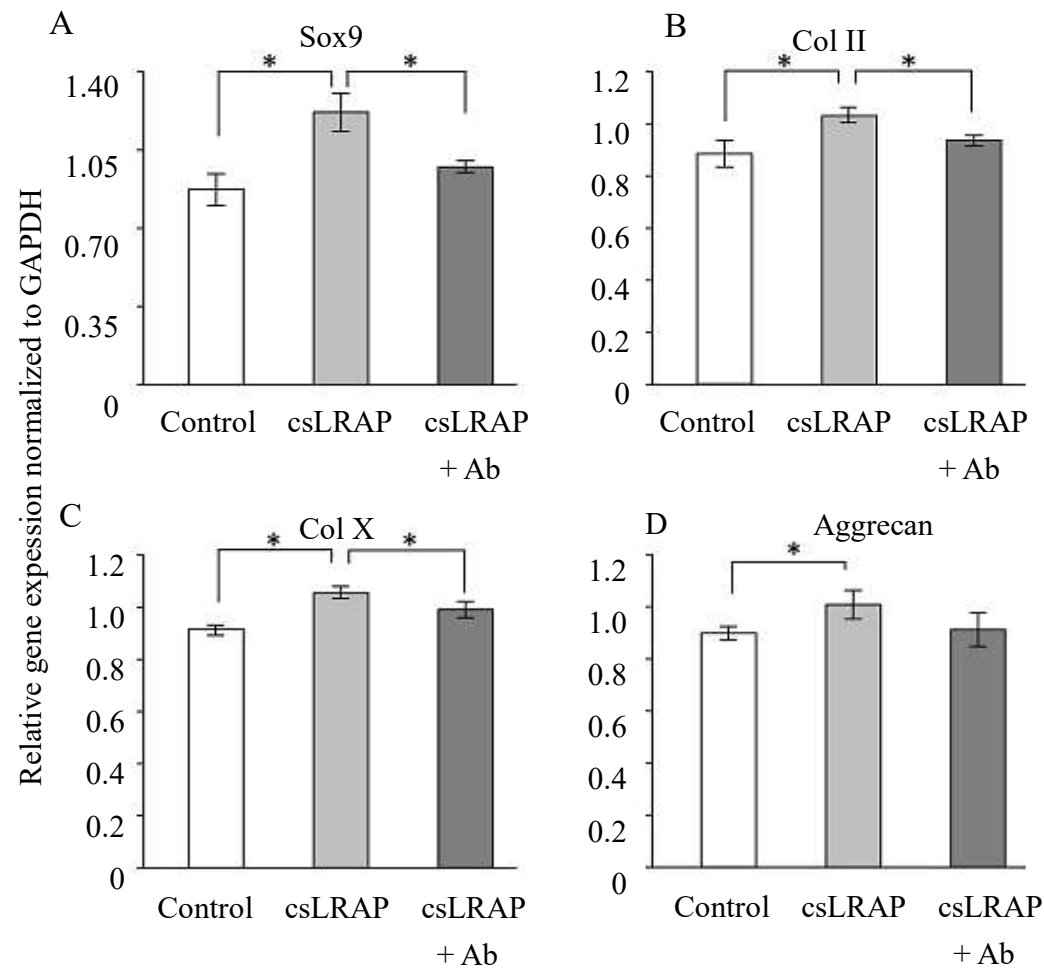

Figure 4. Chondrogenic cells were cultured in chondrogenic differentiation medium containing $10 \mu \mathrm{g} / \mathrm{ml}$ csLRAP in the absence (csLRAP) or presence of LAMP-1 antibody (csLRPA $+\mathrm{Ab}$ ) for one week. Real-time PCR was performed on cDNA generated from total RNA, which was isolated from 6 samples for each chondrogenic differentiation marker gene. All the maker genes were upregulated in the presence of csLRAP at $10 \mu \mathrm{g} / \mathrm{ml}$ (A, B, C, and D). In the presence of LAMP-1 antibody, the expression of Sox9 (A, Sox9), Collagen type II (B, Col II), and Collagen type $\mathrm{X}(\mathrm{C}, \mathrm{Col} \mathrm{X})$ was suppressed (csLRAP $+\mathrm{Ab})$ significantly compared to that in the absence of the antibody (csLRAP). * $p<0.05$

increased significantly compared to that in the control, which was treated only with the chondrogenic differentiation supplement (Fig. 4, Control). Addition of LAMP-1 antibody to the cell culture medium during the week long culture (Fig. 4, csLRAP $+\mathrm{Ab}$ ) resulted in significant suppression of this increased of chondrogenic marker gene expression with the exception of aggrecan, compared to treatment with $10 \mu \mathrm{g} / \mathrm{ml}$ csLRAP alone (Fig. 4D, csLRAP)
Similarly, in the presence of $10 \mu \mathrm{g} / \mathrm{ml}$ csLRAP, all the osteogenic differentiation marker genes including Runx2 (Fig. 5A), ALP (Fig. 5B), Col1 $\alpha 2$ (Fig. 5C), BSP (Fig. 5D), and OPN (Fig. $5 \mathrm{E})$ were showed significantly increased expression compared to that in the control including only the osteogenic differentiation supplement (Fig. 5, Control) after one week of culture. However, OCN (Fig. 5F) showed no significant difference even after 2 weeks of culture (data not shown). Upon addition of LAMP-1 antibody 
A

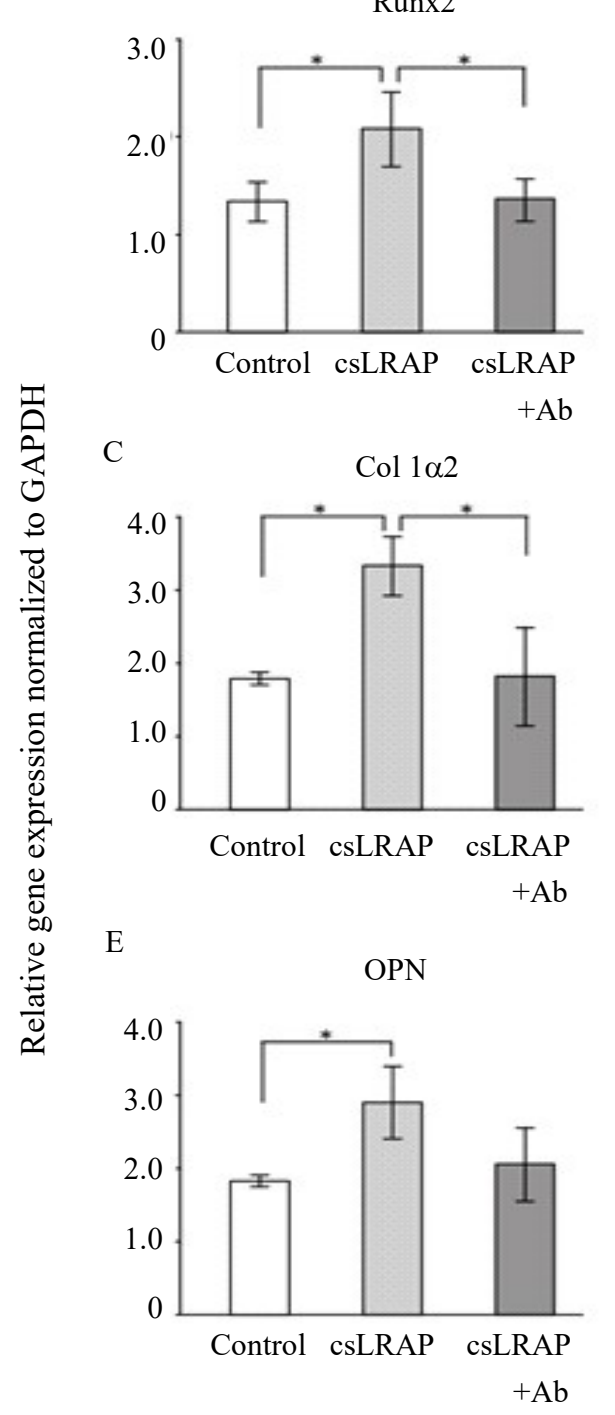

$\mathrm{B}$

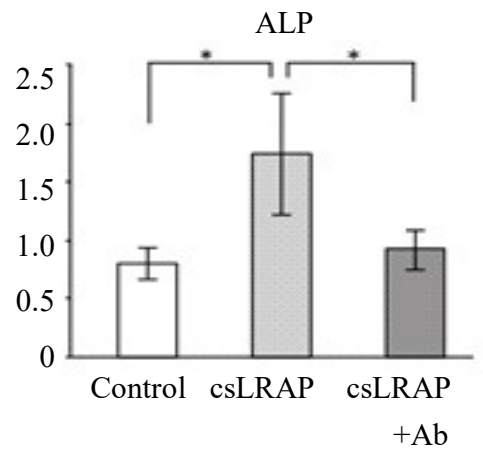

$\mathrm{D}$
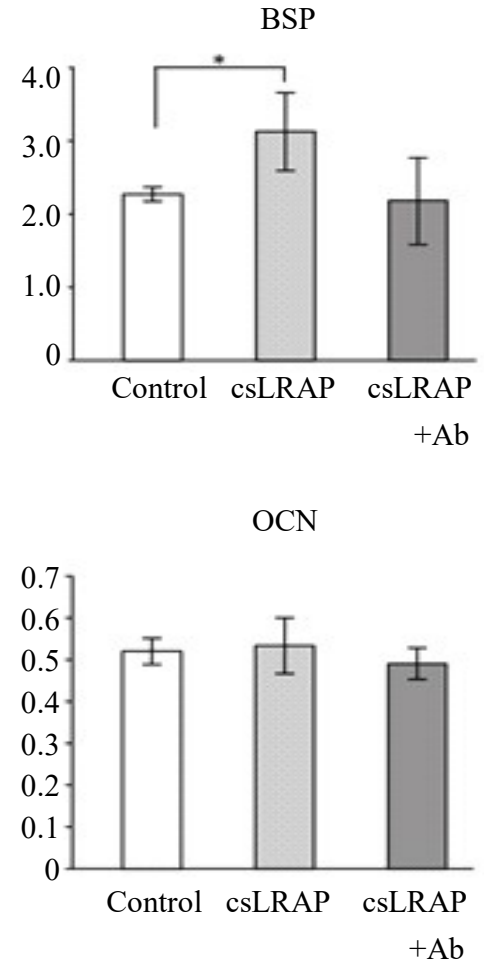

Figure 5. Osteoblast cells were cultured with osteogenic differentiation medium containing $10 \mu \mathrm{g} / \mathrm{ml} \mathrm{csLRAP}$ in the absence or presence of LAMP-1 antibody for one week. Real-time PCR was performed on cDNA generated from total RNA, which was isolated from 6 samples for each osteogenesis differentiation marker gene. All the marker genes except for osteocalcin $(\mathrm{F}, \mathrm{OCN})$ were upregulated in the presence of csLRAP (csLRAP). Increasing Runx2 (A), Alkaline Phosphatase (B, ALP), and Collagen type I alpha 2 (C, Col1a2) expression promoted by csLRAP was significantly suppressed in the presence of LAMP-1 antibody (csLRAP+Ab). ${ }^{*} p<0.05$

A

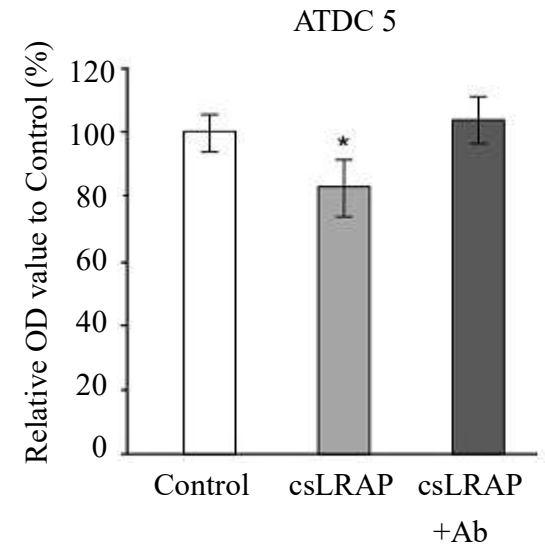

B

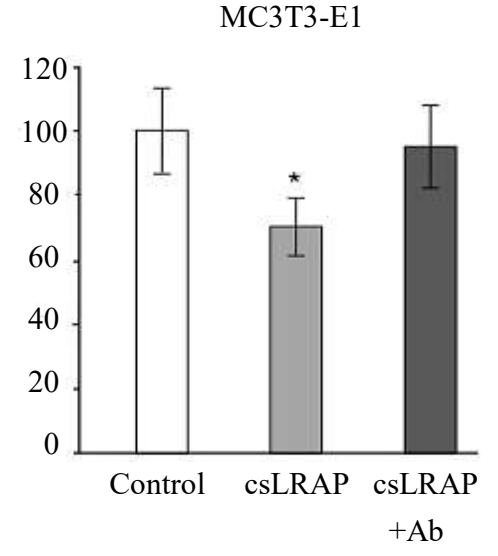

Figure 6. Chondrogenic cells ATDC5 (A) and osteoblastic cells MC3T3-E1 (B) were cultured in respective growth media without serum, with $10 \mu \mathrm{g} / \mathrm{ml}$ csLRAP, in the absence (csLRAP) or presence of LAMP-1 antibody (csLRAP $+A b)$ for 48 hours. After cell culture, the cells were incubated in cell counting medium and the proportional cell numbers were determined by measuring absorbance. The OD value of control (Control), in the absence csLRAP and LAMP-1 antibody, was considered as $100 \%$. In the presence of csLRAP, the cell number of both ATDC5 (A, csLRAP) and MC3T3-E1 (B, csLRAP) was suppressed and the addition of LAMP-1 antibody (A and $B$, csLRAP $+A b$ ) rescued the suppression of cell proliferation induced by csLRAP. $n=16, * p<0.05$ 
Yuko Matsuda et al.: Effects of csLRAP on Chondrogenesis and Osteogenesis

to the cell culture medium during the week long culture (Fig. 5, csLRAP $+\mathrm{Ab}$ ), the increased marker gene expression was significantly suppressed compared to that in the presence of 10 $\mu \mathrm{g} / \mathrm{ml}$ csLRAP alone (Fig. 5, csLRAP). This was observed with Runx2 (Fig. 5A), ALP (Fig. 4B), and Coll $\alpha 2$ (Fig. 5C) and showed decreasing tendency in BSP (Fig. 5D) and OPN (Fig. 5E) although not significantly different.

\section{Cell Number}

In order to systematically investigate the involvement of LAMP-1 in the regulation of chondrogenesis or osteogenesis promoted by csLRAP, we analyzed its effect on cell proliferation. Total cell number in chondrogenic or osteogenic cells was determined after 48 hours of culture in the presence of csLRAP or in the combined presence of csLRAP and the LAMP-1 antibody. Relative cell number was determined by measuring the absorbance of the formazan dye product formed in each culture. Both in chondrogenic cells (Fig. 6A) and in osteogenic cells (Fig. 6B), csLRAP treatment (Fig. 6, csLRAP) significantly suppressed the total cell number compared to that in the absence of csLRAP (Fig. 6, Control); this suppression of cell number was rescued by the presence of the LAMP-1 antibody (Fig. 6, csLRAP + Ab).

\section{Discussion}

Since discovering the effect of amelogenin on chondrogenesis and osteogenesis in vitro ${ }^{4)}$ and the expression of the amelogenin binding protein, LAMP-1, on the cell membrane ${ }^{20)}$, various studies have suggested that amelogenin splice variants including LRAP may participate as signaling molecules in sequential mesenchymal cell differentiation toward the chondrogenic or osteogenic tissue forming cells ${ }^{12-16)}$. However, the mechanisms underlying the effect of LRAP in chondrogenesis and osteogenesis are still unclear. Because the LRAP used in previous studies was generated in biological sources like bacteria or mammalian cells, we produced csLRAP using solid phase synthesis, which yields a protein with higher purity than that by using biological materials ${ }^{23)}$, and then tested the effect of csLRAP in chondrogenic and osteogenic cell differentiation along with clarifying the role of LAMP-1.

In chondrogenic gene expression, csLRAP was found to increase the expression of all genes after one week of culture and these results were similar to those of a previous report using same cell line and culture period, in which Sox9, Col II and aggrecan genes were increased after one week culture ${ }^{13)}$. Thus the chemically synthesized csLRAP may possess a similar biological effect on chondrogenic differentiation as well as recombinant mouse LRAP produced in human embryonic kidney cells. Using mesenchymal cells in which LRAP at $10 \mathrm{ng} / \mathrm{ml}$ increased the expression of chondrogenic marker genes by 48 hours of culture ${ }^{16)}$ suggests that LRAP might have more potent effects on immature cells. Indeed, it has been suggested that LRAP indices osteoinductive molecules which are equal to or more potent than BMP-2 in mouse embryonic stem cells ${ }^{12)}$. Using alcian blue staining, we demonstrated that csLRAP at $10 \mu \mathrm{g} / \mathrm{ml}$ increased the alcian blue staining intensity after 4 weeks of culture. We have previously shown that porcine recombinant LRAP generated from $E$. coli at 1-10 ng/ml, promoted alcian blue staining in mouse limb mesenchyme micromasses after 4 to 6 days of culture ${ }^{16)}$. On the other hand, a previous study showed that high concentrations of recombinant mouse LRAP produced in human embryonic kidney cells promoted alcian blue staining in ATDC5 cells after 21 days of culture ${ }^{13)}$. Furthermore a recent study indicated that the chemically synthesized tyrosinerich amelogenin peptide (TRAP), which is one of the major amelogenin fragments, promoted alcian blue staining in a pellet culture of human articular cartilage cells after 3 weeks at a dose of $10 \mu \mathrm{g} / \mathrm{ml}^{15}$. These studies indicate that csLRAP might enhance the alcian blue staining intensity in chondrogenic cells along with promoting chondrogenic gene expression, although in long term cultures higher doses may be required than using the LRAP generated from biological source like bacteria or mammalian cells.

Similarly, alizarin red staining in MC3T3-E1 cells was increased after 4 weeks of culture with csLRAP at $10 \mu \mathrm{g} / \mathrm{ml}$. This result is similar to that of a previous report, wherein recombinant mouse LRAP increased in vitro calcification in MC3T3-E1 cells as indicated by alizarin red staining after 20 days of culture ${ }^{13)}$. In MC3T3-E1 cells ALP is deeply related to calcification and nodule formation with collagen fiber accumulation and accompanying gene expression $^{31,32)}$. Furthermore the expression of BSP and OCN genes play an important role in the mineralization of MC3T3-E1 cells ${ }^{33)}$, and Runx 2 is the transcriptional regulator of $\mathrm{OPN}^{34)}$ and $\mathrm{OCN}^{35)}$. In our result, although OCN expression was not significantly different from that in a 1-2 week culture, ALP gene expression was increased after one week of culture in the presence of csLRAP at $10 \mu \mathrm{g} / \mathrm{ml}$ and so were Runx2, BSP, Col1a2, and OPN. Several previous studies have suggested that amelogenin peptides corresponding to LRAP were increased along with bone matrix proteins such as BSP and $\mathrm{OPN}^{4,13,36)}$. Using recombinant mouse LRAP produced in human embryonic cells increased Runx2 and OPN gene expression was observed in MC3T3-E1 cells, but no effect was observed on BSP expression after 20 days of culture $^{13)}$. Calcification nodules formation was increased with increasing ALP, BSP and Col1a2 gene expression in the presence of csLRAP. Since recombinant porcine LRAP generated from E.Coli suppressed the gene expression of OCN but promoted OPN in cementoblasts, which form mineralized tissue similar to bone ${ }^{36)}$, csLRAP may have no effect on OCN gene expression.

Previous studies have reported that recombinant full-length amelogenin (rh174 or rm180) binds to LAMP-1 or LAMP-3 expressed on the cell membrane of cementoblasts ${ }^{21,25)}$, and that the presence of LAMP-3 antibody in the culture medium inhibited the cementoblast proliferation promoted by rh $174^{25)}$. Furthermore 
in cementoblast, LRAP showed no effect on cell proliferation ${ }^{36}$. On the other hand our results showed that the presence of LAMP1 antibody in cell culture medium rescued cell proliferation inhibition in the chondrogenic ATDC5 cells expressing LAMP-1, consistent with a previous report that both M180 and LRAP inhibited ATDC5 cell proliferation even though the expression of LAMP-1 in ATDC5 was not examined ${ }^{14)}$. Also it has been reported that LAMP-1 is specific receptor for LRAP ${ }^{37)}$. These results indicate that csLRAP might have similar effects on chondrogenic cell proliferation involving LAMP-1 protein but may have a different effect on mineralized tissue- forming cells like osteoblasts or cementoblasts. It has been suggested that LAMP-1 involves the enamel epithelial cell differentiation ${ }^{20)}$ and amelogenin gene expression in ameloblast cell line promoted by LRAP ${ }^{38)}$. And recent studies have indicated that LAMP-1 expression could be involved in osteoblast MC3T3 differentiation and the regulation of calcification $^{39)}$. Our results showed that several differentiation marker genes in chondrogenic and osteoblast cells were increased by csLRAP and were suppressed in the presence of LAMP-1 antibody. Our previous study suggested that LAMP-1 is involved in chondrogenic differentiation of mesenchymal cells ${ }^{28)}$. Thus, our present results suggest that LAMP-1 expression in chondrogenic cells and osteoblast cells is involved in the differentiation process promoted by csLRAP. However, further studies are needed to understand the role of LRAP and LAMP-1 in the differentiation of chondrogenic or osteoblast cells.

\section{Acknowledgements}

This work was supported by a Grant-in-Aid for Scientific Research (C) from the Ministry of Education, Culture, Sports, Science and Technology, Japan, to YH (23592726).

\section{Conflict of Interest}

The authors have declared that no COI exists.

\section{References}

1. Simmer JP, Hu CC, Lau EC, Sarte P, Slavkin HC and Fincham AG. Alternative splicing of the mouse amelogenin primary RNA transcript. Calcif Tissue Int 55: 302-310, 1994

2. Papagerakis P, Ibarra JM, Inozentseva N, DenBesten P and MacDougall M. Mouse amelogenin exons 8 and 9: sequence analysis and protein distribution. J Dent Res 84: 613-617, 2005

3. Hu CC, Fukae M, Uchida T, Qian Q, Zhang CH, Ryu OH, Tanabe T, Yamakoshi Y, Murakami C, Dohi N, Shimizu M and Simmer JP. Cloning and characterization of porcine enamelin mRNAs. J Dent Res 76: 1720-1729, 1997

4. Veis A, Tompkins K, Alvares K, Wei K, Wang L, Wang XS, Brownell AG, Jengh SM and Healy KE. Specific amelogenin gene splice products have signaling effects on cells in culture and in implants in vivo. J Biol Chem 275: 41263-41272, 2000

5. Haruyama N, Hatakeyama J, Hatakeyama Y, Gibson CW and Kulkarni AB. Lessons from the Amelogenin Knockout Mice. In: Amelogenins: Multifaceted Proteins for Dental \& Bone Formation \& Repair, ed by Goldberg M, Bentham Science Publishers, 2010, pp25-31, eISBN: 978-1-60805-171-7

6. Haze A, Taylor AL, Blumenfeld A, Rosenfeld E, Leiser Y, Dafni L, Shay B, Gruenbaum-Cohen Y, Fermon E, Haegewald S, Bernimoulin JP and Deutsch D. Amelogenin expression in long bone and cartilage cells and in bone marrow progenitor cells. Anat Rec (Hoboken) 290: 455-460, 2007

7. Oida S, Nagano T, Yamakoshi Y, Ando H, Yamada M and Fukae M. Amelogenin gene expression in porcine odontoblasts. J Dent Res 81: 103-108, 2002

8. Hatakeyama J, Sreenath T, Hatakeyama Y, Thyagarajan T, Shum L, Gibson CW, Wright JT and Kulkarni AB. The receptor activator of nuclear factor-kappa $\mathrm{B}$ ligand-mediated osteoclastogenic pathway is elevated in amelogenin-null mice. J Biol Chem 278: 35743-35748, 2003

9. Nuñez J, Sanz M, Hoz-Rodríguez L, Zeichner-David M and Arzate H. Human cementoblasts express enamel-associated molecules in vitro and in vivo. J Periodontal Res 45: 809814,2010

10. Matsuzawa M, Sheu TJ, Lee YJ, Chen M, Li TF, Huang CT, Holz JD and Puzas JE. Putative signaling action of amelogenin utilizes the $\mathrm{Wnt} /$ beta-catenin pathway. J Periodontal Res 44: 289-296, 2009

11. Viswanathan HL, Berry JE, Foster BL, Gibson CW, Li Y, Kulkarni AB, Snead ML and Somerman MJ. Amelogenin: a potential regulator of cementum-associated genes. J Periodontol 74: 1423-1431, 2003

12. Warotayanont R, Zhu D, Snead ML and Zhou Y. Leucinerich amelogenin peptide induces osteogenesis in mouse embryonic stem cells. Biochem Biophys Res Commun 367: $1-6,2008$

13. Mitani K, Haruyama N, Hatakeyama J and Igarashi K. Amelogenin splice isoforms stimulate chondrogenic differentiation of ATDC5 cells. Oral Dis 19: 169-179, 2012

14. Le MH, Warotayanont R, Stahl J, Den Besten PK and Nakano Y. Amelogenin exon4 forms a novel miRNA that directs ameloblast and osteoblast Differentiation. J Dent Res 95: 423-429, 2015

15. Amin HD and Ethier CR. Differential effects of tyrosinerich amelogenin peptide on chondrogenic and osteogenic differentiation of adult chondrocytes. Cell Tissue Res. 364: 219-224, 2016

16. Hatakeyama J, Hatakeyama Y, Matsumoto N, Haruna C, Morotomi T, Izumi T, Sawa Y, Sasano Y and Anan H. 
Yuko Matsuda et al.: Effects of csLRAP on Chondrogenesis and Osteogenesis

Leucine-rich amelogenin peptide promotes chondrogenesis. Jpn J Conserv Dent 56: 560-569, 2013

17. Saftig $\mathbf{P}$ and Klumperman J. Lysosome biogenesis and lysosomal membrane proteins: trafficking meets function. Nat Rev Mol Cell Biol 10: 623-635, 2009

18. Tompkins K, George A and Veis A. Characterization of a mouse amelogenin [A-4]/M59 cell surface receptor. Bone 38: 172-180, 2006

19. Zou Y, Wang H, Shapiro JL, Okamoto CT, Brookes SJ, Lyngstadaas SP, Snead ML and Paine ML. Determination of protein regions responsible for interactions of amelogenin with CD63 and LAMP1. Biochem J 408: 347-354, 2007

20. Le TQ, Zhang Y, Li W and Denbesten PK. The effect of LRAP on enamel organ epithelial cell differentiation. J Dent Res 86: 1095-1099, 2007

21. Zhang H, Tompkins K, Garrigues J, Snead ML, Gibson CW and Somerman MJ. Full length amelogenin binds to cell surface LAMP-1 on tooth root/periodontium associated cells. Arch Oral Biol 55: 417-425, 2010

22. Clement H, Flores V, Diego-Garcia E, Corrales-Garcia L, Villegas E and Corzo G. A comparison between the recombinant expression and chemical synthesis of a short cysteine-rich insecticidal spider peptide. J Venom Anim Toxins Incl Trop Dis 21: 19, 2015.

23. Lau EC, Simmer JP, Bringas P Jr, Hsu DD, Hu CC, ZeichnerDavid M, Thiemann F, Snead ML, Slavkin HC and Fincham AG. Alternative splicing of the mouse amelogenin primary RNA transcript contributes to amelogenin heterogeneity. Biochem Biophys Res Commun 1188: 1253-1260, 1992

24. Hatakeyama Y, Hatakeyama J, Takahashi A, Oka K, Tsuruga E, Inai $T$ and Sawa Y. The effect of valproic Acid on mesenchymal pluripotent cell proliferation and differentiation in extracellular matrices. Drug Target Insights 5: 1-9, 2011

25. Kunimatsu R, Tanimoto K, Tanne Y, Kamiya T, Ohkuma S, Huang YC, Yoshimi Y, Miyauchi M, Takata T and Tanne K. Amelogenin enhances the proliferation of cementoblast lineage cells. J Periodontol 82: 1632-1638, 2011

26. Hatakeyama Y, Tuan RS and Shum L. Distinct functions of BMP4 and GDF5 in the regulation of chondrogenesis. J Cell Biochem 91: 1204-1217, 2004

27. Hessle L, Johnson KA, Anderson HC, Narisawa S, Sali A, Goding JW, Terkeltaub R and Millan JL. Tissue-nonspecific alkaline phosphatase and plasma cell membrane glycoprotein-1 are central antagonistic regulators of bone mineralization. Proc Natl Acad Sci USA 99: 9445-9449, 2002

28. Hatakeyama Y, Hatakeyama J, Oka K, Tsuruga E, Inai T, Anan $\mathrm{H}$ and Sawa Y. Immunohistochemical study of amelogenin and lysosome-associate membrane proteins
(LAMPs) in Cartilage. Int. J. Morphol 32: 618-626, 2014

29. Lee MK, Choi H, Gil M and Nikodem VM. Regulation of osteoblast differentiation by Nurr1 in MC3T3-E1 cell line and mouse calvarial osteoblasts. J Cell Biochem. 99: 98694, 2006

30. Nagata M, Nuckolls GH, Wang X, Shum L, Seki Y, Kawase T, Takahashi K, Nonaka K, Takahashi I, Noman AA, Suzuki $\mathrm{K}$ and Slavkin HC. The primary site of the acrocephalic feature in Apert Syndrome is a dwarf cranial base with accelerated chondrocytic differentiation due to aberrant activation of the FGFR2 signaling. Bone 48: 847-856, 2011

31. Sudo H, Kodama HA, Amagai Y, Yamamoto S and Kasai S. In vitro differentiation and calcification in a new clonal osteogenic cell line derived from newborn mouse calvaria. J Cell Biol 96: 191-198, 1983

32. Quarles LD, Yohay DA, Lever LW, Caton R and Wenstrup RJ. Distinct proliferative and differentiated stages of murine MC3T3-E1 cells in culture: an in vitro model of osteoblast development. J Bone Miner Res 7: 683-692, 1992

33. Wang D, Christensen K, Chawla K, Xiao G, Krebsbach PH and Franceschi RT. Isolation and characterization of MC3T3-E1 preosteoblast subclones with distinct in vitro and in vivo differentiation/mineralization potential. J Bone Miner Res 14: 893-903, 1999

34. Shen Q and Christakos S. The vitamin D receptor, Runx2, and the Notch signaling pathway cooperate in the transcriptional regulation of osteopontin. J Biol Chem 280: 40589-40598, 2005

35. Jeon MJ, Kim JA, Kwon SH, Kim SW, Park KS, Park SW, Kim SY and Shin CS. Activation of peroxisome proliferatoractivated receptor-gamma inhibits the Runx2-mediated transcription of osteocalcin in osteoblasts. J Biol Chem. 278: 23270-23277, 2003

36. Boabaid F, Gibson CW, Kuehl MA, Berry JE, Snead ML, Nociti FH Jr, Katchburian E and Somerman MJ. Leucinerich amelogenin peptide: a candidate signaling molecule during cementogenesis. J Periodontol. 75: 1126-36, 2004

37. Xu L, Harada $\mathrm{H}$ and Taniguchi A. The effects of LAMP1 and LAMP 3 on M180 amelogenin uptake, localization and amelogenin mRNA induction by amelogenin protein. J Biochem. 144: 531-7, 2008

38. Iacob $\mathrm{S}$ and Veis $\mathrm{A}$. Identification of the functional activity of the [A-4] amelogenin gene splice product in newborn mouse ameloblasts. Bone 42: 1072-9. 2008

39. Rajapakshe AR, Podyma-Inoue KA, Terasawa K, Hasegawa K, Namba T, Kumei Y, Yanagishita M and Hara-Yokoyama M. Lysosome-associated membrane proteins (LAMPs) regulate intracellular positioning of mitochondria in MC3T3E1 cells. Exp Cell Res 331: 211-22, 2015 
J.Hard Tissue Biology Vol. 26(1): 51- 60, 2017 